\title{
The Cause of the Predilection of Babesia gibsoni for Reticulocytes
}

\author{
Masahiro YAMASAKI, Yayoi OTSUKA, Osamu YAMATO, Motoshi TAJIMA and Yoshimitsu MAEDE* \\ Laboratory of Internal Medicine, Department of Veterinary Clinical Sciences, Graduate School of Veterinary Medicine, Hokkaido \\ University, Sapporo 060-0818, Japan
}

(Received 14 January 2000/Accepted 21 March 2000)

\begin{abstract}
This study was conducted to determine why Babesia gibsoni replicates well in reticulocytes. First, B. gibsoni was cultivated in resealed erythrocyte ghosts loaded with either erythrocyte or reticulocyte lysate, and in reticulocyte ghosts loaded with either erythrocyte or reticulocyte lysate. The parasites multiplied well in the erythrocyte or reticulocyte ghosts loaded with reticulocyte lysate compared to the other resealed cells loaded with erythrocyte lysate. Second, the parasites were cultivated in erythrocytes in culture medium supplemented with either erythrocyte or reticulocyte lysate. The parasites multiplied better in reticulocyte lysate-containing cultures than in erythrocyte lysate-containing cultures. Finally, the parasites were cultivated in erythrocytes in culture medium supplemented with glutamate, aspartate, asparagine, glycine, isoleucine, proline, taurine or GSH, which were present in higher concentrations in reticulocytes than in erythrocytes. Supplementation of the culture medium with glutamate and GSH resulted in enhancement of the multiplication of the parasites, while the other amino acids did not enhance the multiplication. These results indicated that the high levels of the multiplication of $B$. gibsoni in reticulocytes are partly due to the high concentrations of glutamate and GSH in reticulocytes.
\end{abstract}

KEY WORDS: Babesia gibsoni, glutamate, GSH, reticulocyte

J. Vet. Med. Sci. 62(7): 737-741, 2000.

We previously reported that Babesia gibsoni parasites invade and multiply better in reticulocytes than in mature cells in vitro [16]. It has also been reported that several species of Babesia parasites such as B. rodhaini, B. microti [20] and $B$. canis [4], have a predilection for reticulocytes, while $B$. hylomisci prefers mature erythrocytes to reticulocytes for multiplication [7]. Young erythrocytes have also been reported to show increased susceptibility to invasion by the malaria parasites Plasmodium falciparum [23], P. berghei [21] and $P$. vivax $[11,13]$.

Reticulocytes differ from mature erythrocytes in the following ways. Reticulocytes are larger and have a great excess of membrane in relation to their contents. The cytoplasm contains ribosomes, polyribosomes and mitochondria. They have a higher concentration of adenosine triphosphate (ATP), reduced glutathione (GSH), amino acids and nucleic acids than mature erythrocytes[13]. These compounds may be essential for multiplication of the parasites. Furthermore, dynamic changes in the reticulocyte membrane occur during maturation of the reticulocytes into mature erythrocytes, e. g., remodeling of the membrane skeleton and cytoskeleton [2], the formation of exosomes as a mechanism for the selective loss of the membrane transferrin receptor, and surface-area reduction [9-10]. For several malaria species, successful invasion of erythrocytes has been reported to be associated with the presence of receptors to which malarial merozoites attach on the erythrocyte membrane $[5,10,21,28]$. It is possible that loss of the receptors for $B$. gibsoni parasites could occur with the aging of erythrocytes. On the other hand, it has been reported that the preference of $P$. vivax for invading

\footnotetext{
* Correspondence to: Maede, Y., Laboratory of Internal Medicine, Department of Veterinary Clinical Sciences, Graduate School of Veterinary Medicine, Hokkaido University, Sapporo 060-0818, Japan.
}

reticulocytes might be due to the fact that $P$. vivax merozoites are incapable of invading mature erythrocytes due to a mature cytoskeleton-protein network that makes the erythrocyte membrane less pliable, while the reticulocyte membrane has a less mature cytoskeleton and a more pliable membrane[13]. Thus, the changes in the reticulocyte membrane might facilitate the invasion of the cells by $B$. gibsoni.

However, the reason why $B$. gibsoni parasites multiply better in reticulocytes than in mature erythrocytes is still unclear. The purpose of the present study was to determine the cause of the preferred multiplication of $B$. gibsoni in reticulocytes.

\section{MATERIALS AND METHODS}

Preparation of host erythrocytes: For the preparation of normal canine erythrocytes, six mongrel normal dogs were used. Whole blood, 5-10 ml, was obtained from each dog in a heparinized syringe. The blood was centrifuged at $900 \times \mathrm{g}$ for $5 \mathrm{~min}$ at room temperature. After removal of the supernatant plasma and buffy coat, packed erythrocytes were washed three times with $10 \mathrm{mM}$ phosphate-buffered saline (PBS, $\mathrm{pH}$ 7.4).

Preparation of Babesia gibsoni: For preparation of B. gibsoni-infected erythrocytes, blood was collected from three experimentally infected dogs with parasitemia rates of 4.3 $12.6 \%$ and washed with PBS as described above.

Preparation of canine reticulocytes: Canine reticulocytes were prepared following the method of Inaba and Maede [8] with some modifications. Five normal dogs weighing about $10 \mathrm{~kg}$ were used. Each dog was bled of about $220 \mathrm{~m} l$ blood once daily from the cervical vein for 3 days. On the third day after bleeding, $120 \mathrm{ml}$ of whole blood was collected in a heparinized syringe. At that time, the reticulocyte count in the peripheral blood from each dog was $5.1-8.5 \%$. The blood was washed with PBS as described above and then resuspended in 
PBS to yield a packed-cell volume (PCV) of about $30 \%$. Reticulocytes were separated from the washed cell suspension using the Percoll discontinuous gradient centrifugation. Two kinds of Percoll solution, 45\% (v/v) and 64.5\% (v/v) Percoll solutions containing $150 \mathrm{mM} \mathrm{NaCl}, 0.1 \%(\mathrm{w} / \mathrm{v})$ bovine serum albumin, and $20 \mathrm{mM} \mathrm{HEPES/TRIS} \mathrm{(pH} \mathrm{7.5)} \mathrm{were} \mathrm{used} \mathrm{for}$ preparation of the discontinuous Percoll gradients. The solutions had specific densities of 1.070 and $1.096 \mathrm{~g} / \mathrm{ml}$, respectively. The erythrocyte suspension was carefully layered over the Percoll gradient and centrifuged at $1,800 \times \mathrm{g}$ for $15 \mathrm{~min}$ at room temperature. The cells were distributed a fraction at the interface of the two Percoll solutions and in a fraction of cells with a specific density under $1.096 \mathrm{~g} / \mathrm{ml}$. These two fractions were collected separately. The former cells largely (84-96\%) consisted of reticulocytes and almost all of the latter were mature erythrocytes. The separated cells were washed with PBS as described above.

Preparation of resealed ghosts containing erythrocyte or reticulocyte lysate: Four types of resealed ghosts were prepared: (a) resealed erythrocyte ghosts loaded with erythrocyte lysate (M [M] cells), (b) resealed erythrocyte ghosts with reticulocyte lysate (M [R] cells), (c) resealed reticulocyte ghosts with erythrocyte lysate (R $[\mathrm{M}]$ cells) and $(\mathrm{d})$ resealed reticulocyte ghosts with reticulocyte lysate (R $[R]$ cells). For preparing erythrocyte and reticulocyte lysates, washed erythrocytes and reticulocytes, respectively, were resuspended in PBS to yield a PCV of $70 \%$. Each suspension was mixed with two volumes of lysing buffer ( $5 \mathrm{mM}$ phosphate-buffer (PB), $1 \mathrm{mM}$ Mg-ATP, $\mathrm{pH} 7.4$ ), and centrifuged at $15,000 \times \mathrm{g}$ for $30 \mathrm{~min}$ at $4^{\circ} \mathrm{C}$. The supernatant was collected and stored at $4{ }^{\circ} \mathrm{C}$ until use. For preparing erythrocyte and reticulocyte ghosts, each erythrocyte and reticulocyte suspension with the PCV adjusted to $70 \%$ with PBS, was dialyzed according to the method of Olson and Kilejian [22]. In the present study, 5 $\mathrm{mM}$ PB was used as lysis buffer. The contents of the dialysis tube were used as ghosts. One volume of ghosts, eight volumes of lysate and one volume of $1.2 \mathrm{M} \mathrm{NaCl}$ were mixed and incubated at $37^{\circ} \mathrm{C}$ for $1 \mathrm{hr}$. After the incubation, the mixtures were washed once with PBS and used as resealed ghosts. The mean cell volume (MCV) and mean corpuscular hemoglobin concentration (MCHC) of the resealed ghosts were calculated using SYSTEM 9000 (HEMATOLOGY SERIES CELL COUNTER; Baker INSTRUMENTS).

Culture of Babesia gibsoni: B. gibsoni were cultivated according to the method of Murase et al. [17] with some modifications. Each type of resealed ghosts and the B. gibsoniinfected erythrocytes prepared as described above were washed three times with $\alpha$-medium (Flow Laboratory, U.S.A.) supplemented with sodium pyruvate $(0.11 \mathrm{mg} / \mathrm{ml})$, glutamine $(0.3 \mathrm{mg} / \mathrm{ml})$, sodium bicarbonate $(2 \mathrm{mg} / \mathrm{ml})$, penicillin $(100$ units $/ \mathrm{m} l)$ and streptomycin $(100 \mu \mathrm{g} / \mathrm{m} l)$. After washing, they were resuspended in culture medium consisting of $60 \% \alpha$-medium and $40 \%$ heat-inactivated dog serum, to yield a PCV of $3 \%$. For cultivation of parasites, the $B$. gibsoni-infected erythrocyte suspension was mixed with nine volumes of each type of resealed ghosts. Then $100 \mu \mathrm{l}$ of each mixed suspension was placed in a well of 96-well flat-bottom microculture plates and incubated at $37^{\circ} \mathrm{C}$ in a humidified atmosphere containing 5\% $\mathrm{CO}_{2}$ for 6 days (MODEL 6100 $\mathrm{CO}_{2}$ INCUBATOR, napco). Every $24 \mathrm{hr}, 60 \mu \mathrm{l}$ of the culture supernatant was removed without disturbing the sedimented ghosts and replaced with an equal volume of the fresh culture medium. One well was used every day to make a thin smear sample. The percentage of parasitemia was calculated by counting the number of parasitized cells per 2,000 cells.

For preparing lysates, reticulocytes and erythrocytes were resuspended in $\alpha$-medium to yield a PCV of $50 \%$, and lysed by three cycles of freezing in liquid nitrogen and thawing at room temperature. After centrifugation at $15,000 \times \mathrm{g}$ for 30 min at $4^{\circ} \mathrm{C}$, the supernatant was collected and filtrated using a $0.45-\mu \mathrm{m}$ pore-size filter. The respective filtrated supernatants were used as the reticulocyte and erythrocyte lysates. The lysate medium consisted of $50 \%$ reticulocyte or erythrocyte lysate, $10 \% \alpha$-medium and 40\% normal dog serum. B. gibsoni was cultivated in intact canine erythrocytes in each of the lysate media as described above.

Some compounds, L-glutamate, L-aspartate, L-asparagine, glycine, L-isoleucine, L-proline, taurine and reduced glutathione (GSH) were added to the culture medium at a concentration of about $5 \mathrm{mM}$ each. B. gibsoni was cultivated in intact canine erythrocytes in each of these culture media as described above.

Measurements of intracellular GSH and amino acids: The concentrations of GSH in canine erythrocytes and reticulocytes were measured according to the method of Beutler [1]. The concentrations of intracellular amino acids in these cells were measured as described previously [13].

Statistics: Statistical analysis was carried out using Student's t-test.

\section{RESULTS}

Multiplication of the parasites in resealed ghosts: In these experiments, four types of resealed ghosts were prepared: resealed erythrocyte ghosts loaded with mature erythrocyte lysate (M[M]cells), resealed erythrocyte ghosts with reticulocyte lysate $(\mathrm{M}[\mathrm{R}]$ cells), resealed reticulocyte ghosts with mature erythrocyte lysate (R[M]cells) and resealed reticulocyte ghost loaded with reticulocyte lysate (R[R]cells). There was no significant difference of $\mathrm{MCHC}$ or $\mathrm{MCH}$ among the different types of resealed ghosts and MCV of R[R]cells was only significantly larger than that of $\mathrm{M}[\mathrm{M}]$ cells (Table 1). When these ghosts were used for cultivation of $B$. gibsoni, parasitemia in both the $M[R]$ and $R[R]$ cultures was signifi-

Table 1. Erythrocyte indices of four types of resealed cells ${ }^{\mathrm{a}}$

\begin{tabular}{llrr}
\hline Cell type & \multicolumn{1}{c}{$\mathrm{MCV}(\mathrm{fl})$} & \multicolumn{1}{c}{$\mathrm{MCH}(\mathrm{pg})$} & \multicolumn{1}{c}{$\mathrm{MCHC}(\%)$} \\
\hline $\mathrm{M}[\mathrm{M}]$ cell & $82.4 \pm 0.416$ & $9.3 \pm 0.351$ & $11.3 \pm 0.351$ \\
$\mathrm{M}[\mathrm{R}]$ cell & $82.6 \pm 4.546$ & $10.7 \pm 1.361$ & $12.9 \pm 0.929$ \\
$\mathrm{R}[\mathrm{R}]$ cell & $91.4 \pm 2.411^{\mathrm{b})}$ & $11.2 \pm 5.724$ & $13.4 \pm 2.658$ \\
$\mathrm{R}[\mathrm{M}]$ cell & $81.4 \pm 0.655$ & $6.2 \pm 2.401$ & $7.6 \pm 2.901$ \\
\hline
\end{tabular}

a) Data are expressed as mean \pm S.D. $(n=3)$.

b) Values are significantly $(\mathrm{p}<0.05)$ higher than those of $\mathrm{M}[\mathrm{M}]$ cells. 


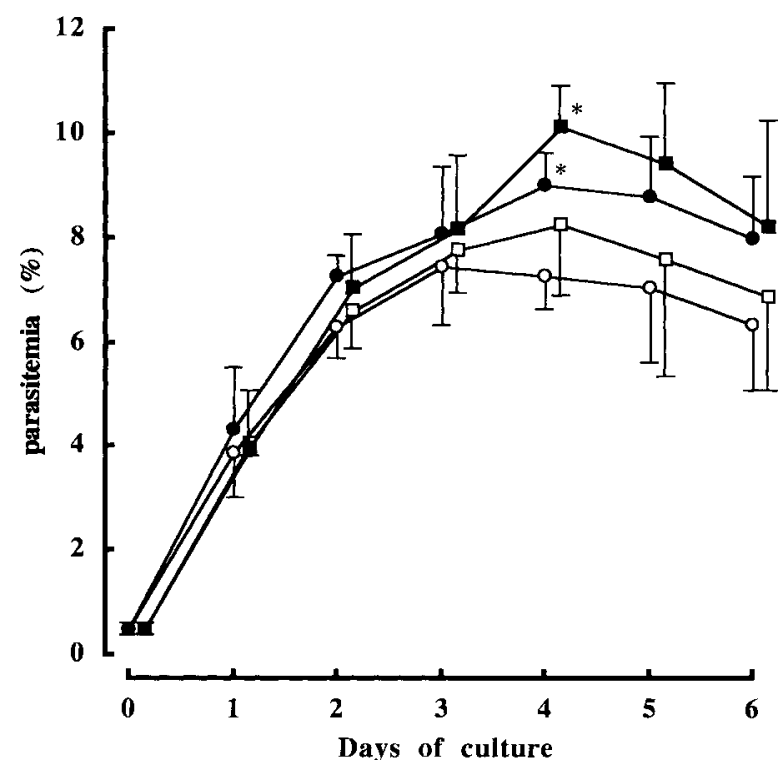

Fig. 1. B. gibsoni was cultivated with $\mathrm{M}[\mathrm{M}](\bigcirc), \mathrm{M}[\mathrm{R}](\mathbf{O}), \mathrm{R}[\mathrm{R}]$ ( $\square$ ) or R[M] ( $\square$ ) resealed ghosts. Data are expressed as mean \pm S.D. $(\mathrm{n}=3)$. * Significantly different from the value for the $\mathrm{M}[\mathrm{M}]$ culture $(\mathrm{p}<0.05)$.

cantly $(\mathrm{p}<0.05)$ higher than in the $\mathrm{M}[\mathrm{M}]$ culture on day 4 , while no significant difference was seen between the R[M] culture and the M[M] culture (Fig. 1).

Effect of lysate on the cultivation of $B$. gibsoni in vitro: $B$. gibsoni was cultivated with canine mature erythrocytes in culture medium with or without $50 \%$ lysate from mature erythrocytes or reticulocytes (Fig. 2). When reticulocyte lysate was added to the culture medium, the level of parasitemia was significantly $(\mathrm{p}<0.05)$ higher than that in the culture without lysate from day 3 to 6 . In contrast, the level of parasitemia in the culture containing mature erythrocyte lysate culture was almost the same as that in the culture without lysate.

Effects of some amino acids and GSH on the replication of B. gibsoni: The concentrations of amino acids and reduced glutathione (GSH) in canine mature erythrocytes and reticulocytes are shown in Table 2. Among them, the concentrations of glutamate, aspartate, asparagine, glycine, isoleucine, taurine, proline and GSH were significantly $(\mathrm{p}<0.05)$ higher in reticulocytes than in mature erythrocytes. When each of these amino acids and GSH were added to the culture medium, a significant $(\mathrm{p}<0.05)$ increase of the parasitemia was observed in the cultures supplemented with L-glutamate or GSH, compared to the control culture (Fig. 3), while the other amino acids had no effect on the multiplication of the parasites (data not shown).

\section{DISCUSSION}

The results of the present study indicate that the preferential of replication of $B$. gibsoni in reticulocytes is due to the intracellular environment of reticulocytes rather than the structure

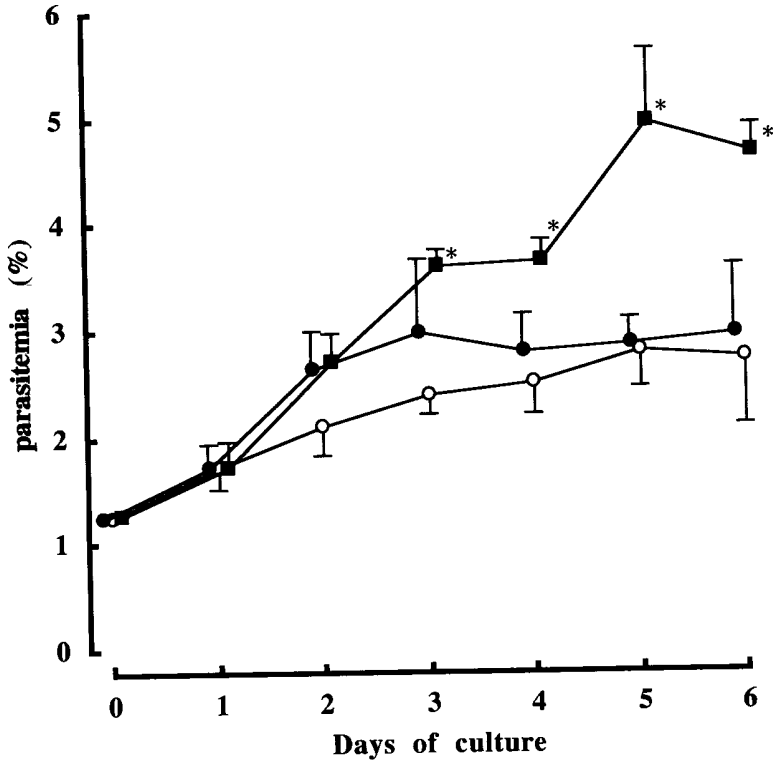

Fig. 2. B. gibsoni was cultivated with canine mature erythrocytes in the presence of erythrocyte ( $)$ or reticulocyte ( $)$ lysate. The cultivation of $B$. gibsoni in canine mature erythrocytes without lysate $(\bigcirc)$ is shown as a control. Data are expressed as mean \pm S.D. $(n=3) . *$ Significantly different from the value for control.

Table 2. The concentrations of amino acids and reduced glutathione (GSH) in canine erythrocytes and reticulocytes ${ }^{\text {a) }}$

\begin{tabular}{lcc}
\hline Substance & Erythrocytes & Reticulocytes \\
\hline alanine & $0.21 \pm 0.010$ & $0.52 \pm 0.203$ \\
arginine & $0.15 \pm 0.009$ & $0.27 \pm 0.104$ \\
aspargine & $0.03 \pm 0.008$ & $0.13 \pm 0.050^{\mathrm{b})}$ \\
aspartate & $0.06 \pm 0.002$ & $0.29 \pm 0.115^{\mathrm{b})}$ \\
glutamate & $0.07 \pm 0.002$ & $0.52 \pm 0.210^{\mathrm{b}}$ \\
glutamine & $0.19 \pm 0.008$ & $0.48 \pm 0.222$ \\
glycine & $0.11 \pm 0.012$ & $0.69 \pm 0.261^{\mathrm{b})}$ \\
histidine & $0.05 \pm 0.001$ & $0.06 \pm 0.030$ \\
isoleucine & $0.04 \pm 0.002$ & $0.13 \pm 0.054^{\mathrm{b})}$ \\
leucine & $0.06 \pm 0.004$ & $0.13 \pm 0.054$ \\
lysine & $0.16 \pm 0.010$ & $0.25 \pm 0.095$ \\
ornithine & $0.04 \pm 0.003$ & $0.04 \pm 0.012$ \\
phenylalanine & $0.04 \pm 0.002$ & $0.10 \pm 0.049$ \\
proline & $0.09 \pm 0.005$ & $0.42 \pm 0.187^{\mathrm{b})}$ \\
serine & $0.11 \pm 0.012$ & $0.22 \pm 0.085$ \\
taurine & $0.18 \pm 0.014$ & $0.59 \pm 0.229^{\mathrm{b})}$ \\
threonine & $0.12 \pm 0.001$ & $0.25 \pm 0.101$ \\
tyrosine & $0.06 \pm 0.005$ & $0.11 \pm 0.054$ \\
valine & $0.08 \pm 0.003$ & $0.15 \pm 0.062$ \\
GSH & $1.16 \pm 0.033$ & $3.28 \pm 0.04^{\mathrm{b})}$ \\
\hline
\end{tabular}

a) nmols $/ 10^{7}$ RBCs. Data are expressed as mean \pm S.D. $(n=3)$.

b) Values are significantly $(\mathrm{p}<0.05)$ higher than those of erythrocyte.

of the cell membrane, because the parasites multiplied better in resealed ghosts loaded with reticulocyte lysate than in ghosts loaded with erythrocyte lysate. Furthermore, the multiplication of $B$. gibsoni was significantly increased when the 


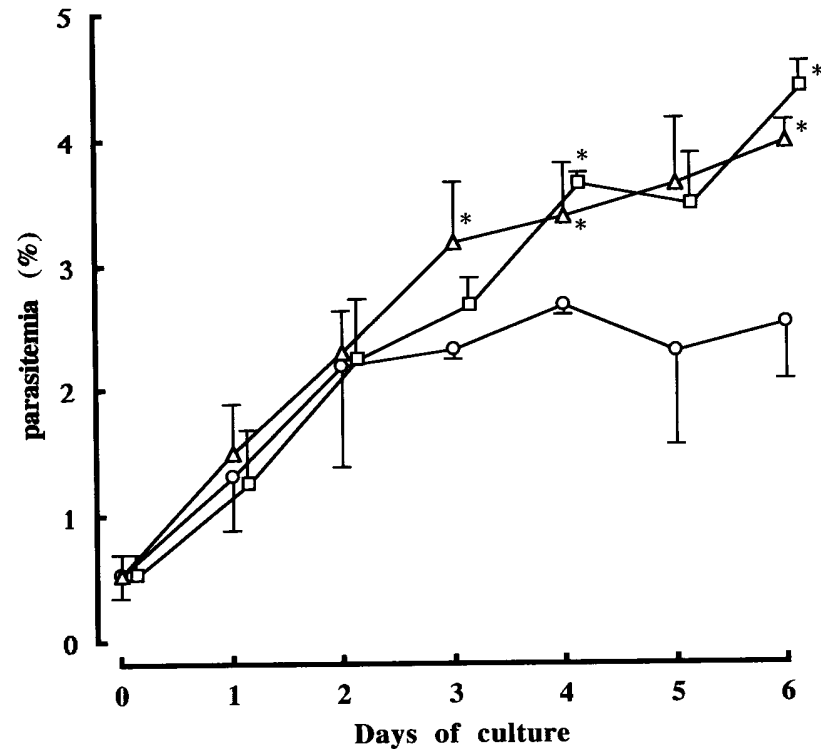

Fig. 3. B. gibsoni was cultivated in erythrocytes in culture medium supplemented with L-glutamate ( $\square$ ) or GSH ( $\triangle$ ). The cultivation of $B$. gibsoni in the culture medium without supplementation $(\bigcirc)$ is shown as a control. Data are expressed as mean \pm S.D. $(n=3)$ * Significantly different from the value for control.

parasite was cultivated in the presence of reticulocyte lysate in the culture medium, while the lysate of mature erythrocytes had no such effect on the parasites. These results suggested that reticulocyte lysate might contain some substance(s) which enhance the growth and multiplication of the parasites in vitro. Biochemical analysis of reticulocyte lysate revealed that reticulocytes contained higher concentrations of several amino acids and GSH than mature erythrocytes. Addition of those substances to the culture medium revealed that the multiplication of the parasites was enhanced by the addition of glutamate or GSH, while the other amino acids had no such effect. These results suggested that the higher concentrations of glutamate and GSH in reticulocytes might be a cause of the predilection of the parasites for reticulocytes. Since the $\mathrm{MCH}$ and $\mathrm{MCHC}$ of all of these types of resealed ghosts were similar, the intracellular hemoglobin concentration seems not to be related to the high level of multiplication of the parasites in reticulocytes.

Since canine erythrocytes possess a $\mathrm{Na}^{+}$-dependent glutamate transport system [8], extracellular glutamate seems to be easily transported into erythrocytes and utilized for the multiplication of the parasites. The avian malarial parasite Plasmodium lophurae is known to contain a glutamate dehydrogenase that is capable of oxidizing glutamate to $\alpha$-ketoglutarate, which suggests that the oxidation of glutamate and its further metabolism via the TCA cycle may provide an energy source for the parasite [26]. Similarly, a glutamate dehydrogenase has been found in erythrocytes infected with the Babesia species such as $B$. bovis, B. bigemina and B. microti $[15,24$, 29 ], while it has not been detected in $B$. gibsoni. Increases of glutamine influx and glutamate formation were observed in erythrocytes infected with a human malaria parasite, $P$. falciparum [25]. Because human erythrocytes are essentially impermeable to glutamate [3], extracellular glutamine might be transported into the cells and converted into glutamate through glutaminase, which is present in P. falciparuminfected erythrocytes [30]. These previous observations and the results of present study indicate that glutamate is an additional energy source for B. gibsoni.

Erythrocyte GSH is believed to participate in intracellular redox reactions and to protect hemoglobin and some of the thiol-dependent enzymes or membrane proteins from oxidative stress. On the other hand, oxidative stress is thought to be a mechanism for the inhibition of malaria parasite growth in vivo [25]. Oxygen radicals would injure not only host erythrocytes but also the parasite harbored in the cells. Indeed, intracellular replication of the malaria parasites in G6PD-deficient erythrocytes that are sensitive to oxidative stress is impaired, especially if these cells are subjected to oxidative stress [19]. Also, those reports suggested that intracellular GSH is an essential substance for protecting the malaria parasite from oxidative stress in the host cells. In the present study, however, it is unclear the reason why extracellular GSH supplemented in the culture medium accelerated the multiplication of the parasites, because GSH can neither enter nor leave erythrocyte [27]. In this respect, we have previously demonstrated that oxidative damage was induced by the multiplication of B. gibsoni in vitro, and that non-parasitized erythrocytes were also exposed to oxidative stress during the parasite cultivation [18]. From these previous findings, it is supposed that the extracellular GSH might protect both of the host cell and the parasite from oxidative stress, resulting in the well multiplication of the parasite [5]. Thus, we conclude that the high concentration of GSH in reticulocytes might protect the parasite from oxidative damage, resulting in the high level of multiplication of B. gibsoni in reticulocytes in vitro.

ACKNOWLEDGMENTS. This work was supported in part by a grant from the Scientific Research Fund of the Ministry of Education, Science, and Culture of Japan. We thank Ms. Hiroko Matsumoto at the Center for Instruments Analysis of Hokkaido University for amino acid analysis.

\section{REFERENCES}

1. Beutler, E. 1984. Reduced glutathione (GSH). pp. 131-134. In: Red Cell Metabolism, Grune \& Stratton Inc., Orlando.

2. Chasis, A. J., Predent, M., Leung, A. and Mohondas, N. 1989. Membrane assembly and remodeling during reticulocyte maturation. Blood 74: 1112-1120.

3. Divo, A. A., Geary, T. G., Davis, N. L. and Jensen, J. B. 1985. Nutritional requirements of Plasmodium falciparum in culture. Exogenously supplied dialyzables components necessary for continuous growth. J. Protozool. 32: 59-64.

4. Eaton, P. 1934. Piroplasm canis in Florida. J. Parasitol. 20: 312-313.

5. Friedman, M. J. 1979. Oxidant damages mediates variant red cell resistance to malaria. Nature (Lond.) 280: 245-247. 
6. Holder, A. A. 1994. Proteins on the surface of the malaria parasites and cell invasion. Parasitology 108: S5-18.

7. Hussein, H. S. 1976. Babesia hylomysci in mice: preference for erythrocytes of a particular age-group and pathogenesis of the anaemia. Z. Parasitenkd. 50: 103-108.

8. Inaba, M. and Maede, Y. 1984. Increase of $\mathrm{Na}^{+}$gradientdependent L-glutamate and L-aspartate transport in high $\mathrm{K}^{+}$ dog erythrocytes associated with high activity of $\left(\mathrm{Na}^{+}, \mathrm{K}^{+}\right)-$ ATPase. J. Biol. Chem. 259: 312-317.

9. Johnstone, R. M., Bianchini, A. and Teng, K. 1989. Reticulocyte maturation and exosome release: Transferrin receptor containing exosome show multiple plasma membrane functions. Blood 74: 1844-1851.

10. Johnstone, R. M., Mathew, A., Mason, A. B. and Teng, K. 1991. Exosome formation during maturation of mammalian and avian reticulocyte: Evidence that exosome release is a major route for externalization of absolute membrane proteins. J. Cell Physiol. 147: 27-36.

11. Kitchin, S. F. 1938. The infection of reticulocytes by Plasmodium vivax. Am. J. Trop. Med. 18: 347-359.

12. Maede, Y. and Inaba, M. 1985. (Na, K)-ATPase and ouabain binding in reticulocytes from dogs with high $\mathrm{K}$ and low $\mathrm{K}$ erythrocytes and their changes during maturation. J. Biol. Chem. 260: 3337-3343.

13. Maede, Y., Kasai, N. and Taniguchi, N. 1982. Hereditary high concentration of glutathione in canine erythrocytes associated with high accumulation of glutamate, glutamine, and aspartate. Blood 59: 883-889.

14. Mons, B. 1990. Preferential invasion of malarial merozoites into young red blood cells. Blood Cells 16: 299-312.

15. Moss, D. M., Heary, G. R., Dickerson, J. W. and Mathews, H. 1986. Isoenzyme analysis of Babesia microti infections in humans. J. Protozool. 33: 213-215.

16. Murase, T., Iwai, M. and Maede, Y. 1993. Direct evidence for preferential multiplication of Babesia gibsoni in young erythrocytes. Parasitol. Res. 79: 269-271.

17. Murase, T., Hashimoto, T., Ueda, T. and Maede, Y. 1991. Multiplication of Babesia gibsoni in in vitro culture and its relation to hemolysis of infected erythrocytes. J. Vet. Med. Sci. 53: 759-760.

18. Murase, T., Ueda, O., Yamato, O., Tajima, M. and Maede, Y. 1996. Oxidative damage and enhanced erythrophagocytosis in canine erythrocytes infected with Babesia gibsoni. J. Vet.
Med. Sci. 53: 259-261.

19. Nagel, R. L. and Roth, E. F. 1989. Malaria and red cell genetic defects. Blood 74: 1213-1221.

20. Nowell, F. 1969. The blood picture resulting from Nuttallia (= Babesia) rodhaini and Nuttallia (=Babesia) microti infections in rats and mice. Parasitology 59: 991-1004.

21. O’Donovan, S. and Dalton, J. P. 1990. Identification of a putative Plasmodium berghei (a rodent malaria parasite) reticulocyte receptor. Biochem. Soc. Trans. 18: 338-339.

22. Olson, J. A. and Kilejian, A. 1982. Involvement of spectrin and ATP in infection of resealed erythrocyte ghosts by the human malarial parasite, Plasmodium falciparum. J. Cell Biol. 95: 757-762.

23. Pasvol, G., Weatherall, D. J. and Wilson, R. J. M. 1980. The increased susceptibility of young red cells to invasion by the malarial parasite. Br. J. Heamatol. 45: 285-295.

24. Rodriguez, G. M., Buening, G. M., Vega, C. A. and Carson, C. A. 1986. Enzymatic characterization of Babesia bovis. J. Protozool. 33: 507-511.

25. Roth, E. F. Jr., Schulman, S., Vanderberg, J. and Olson, J. 1986. Pathway for the reduction of oxidized glutathione in the Plasmodium falciparum-infected erythrocyte: Can parasite enzymes replace host red cell glucose-6-phosphate dehydrogenase? Blood 67: 827-830.

26. Sherman, I. W., Peterson, I., Tanigoshi, L. and Ting, I. P. 1971. The glutamate dehydrogenase of Plasmodium lophurae (avian malaria). Exp. Parasitol. 29: 433-439.

27. Srivastava, S. K. and Beutler, E. 1969. The transport of oxidized glutathione from human erythrocytes. J. Biol. Chem. 244: 9-16.

28. Urquiza, M., Rodriguez, L. E., Suarez, J. E, Guzman, F., Ocampo, M., Curtidor, H., Segura, C., Trujillo, E. and Patarroyo, M. E. 1996. Identification of Plasmodium falciparum MSP-1 peptides able to bind to human red blood cells. Parasite Immunol. 18: 515-526.

29. Vega, C. A., Buening, G. M., Rodriguez, G. M. and Carson, C. A. 1986. Concentration and enzyme content of in vitro-cultured Babesia bigemina-infected erythrocytes. J. Protozool. 33: 514-518.

30. Vilmont, M., Azoulay, M. and Frappier, F. 1990. Metabolism of glutamine in erythrocytes infected with the human malarial parasites: Plasmodium falciparum. Ann. Parasitol. Humm. Comparee. 65: 162-166. 